\title{
DESCRIPTION OF THE DISINTEGRATING FLOUR MILL, AND MACHINE FOR PULVERISING MINERALS \&c., WITHOUT GRINDING, CRUSHING, OR STAMPING.
}

By Mr. THOMAS CARR, OF BRTSTOL.

The object of the writer in designing this machine was to obtain the means of pulverising unfibrous materials of different kinds, without subjecting them to friction or compression, one or both of which objections appertain more or less to all other methods hitherto used for the reduction of materials : such as the processes of grinding, crushing, or stamping, where the material intervenes between two acting surfaces, by which it is held or supported at the time of being operated upon. The writer was led to design the disintegrating machine in consequence of practically experiencing the difficulty and imperfection attending the mode of pulverising artificial manures, such as superphosphate of lime, by the means then available for the purpose. Owing to its containing some combined moisture, this material is liable to get into a pasty condition when crushed; but he found that when a lump of it was struck a rapid blow by a stick, whilst thrown up in the air, it became completely shattered, bursting into minute fragments as though under the action of some explosive force. The way in which this result is effected in the disintegrator is by causing the pieces of the material to fly through the machine with the projectile impetus communicated to them by the centrifugal force resulting from rapid rotation; and these flying pieces are struck in mid air with reiterated blows, delivered with extreme rapidity in alternately opposite directions by a succession of rotating beaters, and are thus shattered by collision against the unarrested beaters, which encounter them in the opposite direction to that in which they were moving. As the particles struck can offer no resistance but 
that which is due to their own inertia, without the aid of any solid abutment to support them when receiving the blows, no friction or compression is produced, and the moving power of the beaters is not needlessly neutralised and absorbed by any such unyielding abutment.

The disintegrating mills, which were originally designed for granulating superphosphate of lime when it had conglomerated into a pasty mass after being partially dissolved by vitriol, were subsequently applied successfully to granulating clays, ores, and other minerals. As however the recent adaptation of the disintegrator to a flour mill forms by far the most important application, this will here be described first.

The Disintegrating Flour Mill is shown in Figs. 1 to 3, Plates 1 and 2 ; Fig. 1 is an external side elevation of an entire machine, 7 feet diameter; Fig. 2 a-transverse section taken through the centre of the machine; and Fig. 3 a longitudinal section of the two rotating dises with a portion of their respective shafts; Fig. 4, Plate 3, shows a portion of the transverse section to a larger scale.

The machine consists of a pair of circular dises $A$ and $B$, Fig. 1, rotating in contrary directions upon two shafts $\mathrm{D}$ and $\mathrm{E}$ situated in the same line; the opposing faces of the discs are studded with a series of short projecting bars or beaters, arranged in successive concentric rings or cages; and the rings of beaters fixed in one disc intervene alternately between those fixed in the other disc, and revolve in the opposite direction. The solid cireular disc A, keyed on the left-hand shaft D, as shown in Fig. 3, carries the third cage or ring of beaters (counting outwards from the centre), and also the fifth, seventh, ninth, and eleventh cages, all of which therefore rotate the same way. On the right-hand shaft $\mathrm{E}$ is keyed a small inner disc $\mathrm{C}$, into which are riveted the bars of the two invermost cages of beaters, their other ends being riveted into the right.hand annular disc $\mathbf{B}$, which is thus carried by them; this annular disc in turn carries the fourth, sixth, eighth, and tenth cages, which with the two innermost all rotate in the contrary direction to the cages carried by the left-hand disc A, as indicated 
by the arrows in Fig. 4. The two innermost cages are both fixed in the same disc so as to rotate both in the same direction, in order thereby to ensure distributing the material more effectually through the machine by the centrifugal force. The cages of beaters are of successively increasing diameters, and consist of $\frac{1}{2}$ inch round steel bars, with clear spaces between of about 2 inches in each direction; the outer ends of the bars in each cage are tied together by a ring, as shown in Figs. 2 and 3, and to a larger scale in Fig. 9, Plate 6.

The two shafts $D$ and $E$ are placed in a line, their rounded ends just touching each other, or nearly so, in the centre, Fig. 9; everywhere else ample clearance is allowed for enabling the two halves of the machine to rotate entirely independent, acting only in unison as auxiliary to each other in pulverising the material that is being operated upon. The shafts are each mounted in two plummer blocks on a heavy square bedplate; and a driving pulley is keyed either in the middle of each shaft or at its outer end, as may be found most convenient for the driving straps, one of which is a crossed strap and the other an open one, so as to drive the two balves of the machine in opposite directions. The revolving cages of beaters are enclosed within an external casing F, Figs. 1 to 3, which has a centre opening in the right-hand side, corresponding with that of the annular dise $B$.

The grain is delivered down a fixed shoot G, Figs. 1 and 3, through the centre opening of the outer casing, into the innermost cage, from which it is instantly projected through the machine, and delivered in a radiating shower from every portion of the circumference into the outer casing, in the form of a meal, similar to that thrown out by the ordinary millstones; to this state the grain is reduced almost instantaneously by being dashed to the right and left alternately by the bars of each of the successive cages revolving in opposite directions at a very high speed. As it falls to the bottom of the casing, the meal is continuously removed by the ordinary rotating screw H, Fig. 2, used in flour mills; it is then passed through the usual bolting machines to separate the bran, and subsequently through silk dressing-machines to separate the fine flour from the semolina. The latter is then winnowed by an exhaust 
current of air in a machine for that purpose, so as to free it from all finely powdered bran, and is afterwards ground between millstones, of which three or four pairs are kept for the purpose; the flour resulting from it is added to the fine flour produced at the outset by the disintegrating flour mill, and to ensure perfect intermixture the two are then passed through the silk dressing-machines together.

The course of a particle through the disintegrator is illustrated in the diagram, Fig. 5, Plate 3 ; the circular arrows show the reverse direction in which the alternate cages rotate, and the straight arrows at different angles show the zigzag course of a particle of material as it flies off at a tangent from each cage, being struck alternately to the right and left and projected thereby at a speed equivalent to that at which the bars of the cage last striking it were rotating; the force of each blow is thus increased by the momentum of the material, which is moving in each case in an opposite direction to that of the beaters it next meets with. As the material becomes more finely pulverised in its course outwards through the machine, and the particles have consequently less inertia of themselves to act as an abutment for receiving the blows of the beaters, a greater force of blow is necessary, in order to continue the pulverising process. This increased force is supplied by the higher velocity arising from the larger diameters of the successive rings of beaters which the material meets with in its passage outwards. The machine is diriven at a speed of about 400 revolutions per minute; and the outermost ring being $6 \mathrm{ft} .10$ ins. diameter, the last beaters have a velocity of 140 feet per second, or about 100 miles per hour; this is double the velocity and consequently gives four times the force of blow of the innermost ring of beaters, the force of blow being proportionate to the square of the velocity.

In this mode of action, by the free blows of the beaters upon the material, the friction and compression between the machine and the material, which are involved in all grinding, crushing, or stamping processes, are avoided, this mill being the only machine that does not act upon the material between a pair of surfaces; and as the beaters do not strike upon any solid abutment, the whole power 
employed is usefully expended in pulverising the material, excepting only the portion of the power absorbed by the resistance of the air to the rotation of the beaters. This mill has the advantage of unusual freedom from risk of injury by the accidental introduction of any unsuitable substances, such as pieces of metal; any such substances are freely ejected by the centrifugal force, without the possibility of any squeezing action being exerted upon them. The machine has not any tendency to become choked, nor are any working parts liable to get out of order, as the two sets of beaters revolve entirely clear of each other, and the beaters never come in contact with anything but the free particles of the material that is being pulverised. In the case of the flour mill, the beaters being of steel, and coming in contact only with the grains of wheat, are not subjected to any perceptible wear, and keep at work continuously without ever requiring any dressing or attention. But with the ordinary millstones, a surplus supply of stones, amounting to oneeighth of the whole number, has always to be kept out of work, to allow for the dressing and sharpening which is usually required to be done upon each pair of stones after about every four days' work.

Two of these disintegrating flour mills are in regular work for 2.2 hours per day at the Bonnington Mills of Messrs. Gibson and Walker at Edinburgh, and have proved completely successful during a year's continuous work. The percentage of flour produced from the same wheat is practically the same as with millstones; but the quality of the flour is found to be decidedly superior, in consequence of the freedom from the compressing action that accompanies grinding by millstones. The flour is delivered in a finely granular state, without being "killed," as it is termed, or rendered dense and partly impervious to water by too fine grinding under frictional pressure; it consequently absorbs more water when used, and is of the quality esteemed for baking into a lighter and better-keeping bread. The bran is also more perfectly separated from the flour in the subsequent dressing process, in consequence of having been scaled off from the grain in larger flakes and less broken up than in grinding by stones. 
The work regularly got through by each machine of 7 feet diameter amounts to 20 quarters of wheat or 160 bushels per hour; which would require as many as twenty-seven pairs of ordinary millstones in full work, taking the average duty of each at 6 bushels per hour. A further supply of three or four pairs of stones under the dresser's hands would be required for keeping that number at work; but these are compensated for by the three or four pairs of finishing stones which are used with the disintegrating mill for grinding the granular portion called semolina, as before explained.

The ultimate result with the disintegrating mill at Edinburgh, when working upon Scotch wheat alone, after the fine flour produced direct from the mill has been mixed with the ground semolina, is $57 \frac{1}{2}$ per cent. of finest flour, worth $5 s$. per sack more than flour from the same wheat ground by millstones. The latter however give a total of 68 per cent. of fine flour, but of less value by $5 s$. per sack. The remainder of the flour from the disintegrator is $10 \frac{1}{2}$ per cent. of an inferior quality, worth from $5 s$. to $10 s$. per sack less than best millstone flour; and taking this at its lowest value, the following is the comparative total value of the flour obtained from 100 sacks of the same wheat by the two processes :-

With the Disintegrator

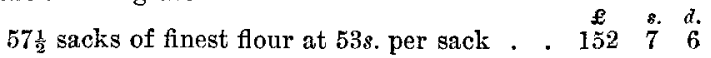

1013 inferior $" 38 s . \quad, \quad$. . 19190

With Millstones

Total. . $\overline{172 \quad 6 \quad 6}$

68 sacks of fine flour at $48 s$. per sack . . . 16340

Difference $£ \longdiv { 9 \quad 2 \quad 6 }$

There is thus a difference in favour of the disintegrator of $£ 9$, or $5 \frac{1}{2}$ per cent. in the item of marketable value of the flour. This gain is equivalent to an extra profit of $£ 16$ on each 100 quarters of wheat (a sack being $280 \mathrm{lbs}$. and a quarter $496 \mathrm{lbs}$ ) ; and the rate of production being 20 quarters per hour, it will be seen that the annual value is a very important amount.* In addition there is the saving in power and labour employed, in

* The profit would be less in amount on the harder and drier qualities of wheat, which are higher priced than the softer Scotch wheat. 
space occupied, and in cost of repairs; and as regards the last item, the repairs have been practically nothing with the disintegrating flour mill during the twelve months' constant work of 22 hours per day, whilst with millstones a heavy cost is constantly incurred in stone dressing.

The disintegrating flour mills at present in use are made with fourteen rotating cages, as shown in Fig. 9, Plate 6 , instead of only eleven cages, as in Figs. 1, 2, and 3; but the fourteen have been found to be more than are necessary, while one mill also in use that has only eight is found scarcely sufficient. The beaters are also made much shorter now than those hitherto used, being only 3 inches long in the clear, as in Fig. 3, instead of 8 inches as in Fig. 9, in order to bring the discs so much nearer together, and thereby diminish proportionately the loss of power in churning the air, which was found in the experiments made at Edinburgh to be more serious than had been at all anticipated. The capacity of the machine with the. reduced width will still be far beyond the requirements, when operating on only 20 quarters of wheat per hour; for the velocity of the material in passing through the mill is so great that a mere fraction of a second elapses from entrance to exit of any given particle, and hence there can never be more than a few handfuls of the material in the machine at any one instant. In other new machines at present making, the bars being now but little more than mere pegs, the tie rings at their extremities are dispensed with, as shown in Fig. 10, Plate 7, being no longer necessary for so light and small a material as wheat. By the omission of these tie rings the successive circles or cages of beaters can be placed much nearer the circumference, whereby their respective diameters and consequently their speeds in feet per second are proportionately increased. The machine is remarkable for its simplicity of construction and non-liability to deteriorate in efficiency in consequence of wear, and for its large production and the superiority of its work; and also for the very small space it occupies, in comparison with that taken up by the twenty-seven pairs of ordinary millstones which are required to perform the same amount of work. 
The Mineral Disintegrator, of which the flour mill was constructed as a modification, is shown in Figs. 6 to 8, Plates 4 and 5 , and is used for pulverising various mineral substances, such as artificial manures, zinc ores, rock asphalte, \&c. It is made of much greater strength in the beaters than the flour mill, and of smaller diameter, being only $4 \frac{1}{2}$ feet instead of 7 feet diameter, and having only fonr cages of beaters instead of eleven or fourteen. The two discs are both carried from the same side of the machine, the shaft of the left-hand one being made tubular, and that of the right-hand disc carried through it without touching it; as shown in Fig. 6 ; by this arrangement the central opening through which the material is fed into the machine is left entirely unobstructed by the driving straps, and the material can be thrown into it by a shovel. This machine is not so well adapted for high speeds of working as the flour mill, on account of the great size of the bearings of the hollow shaft. The speed it is driven at varies from 350 to 500 revolutions per minute, according to the hardness of the material that is being pulverised, the degree of fineness to which it has to be reduced, and the driving power available. A larger size of the machine is made, of $6 \frac{1}{4}$ feet diameter, and also a smaller size of $3 \frac{1}{2}$ feet diameter.

When a soft and adhesive material is operated upon, a portion adheres to each beater, and the machine sometimes, though very rarely, requires cleaning after ten or twelve hours' working. As the material adheres only to the back surface of each bar, while the front remains clean, the machine is readily cleaned by running it backwards for a short time, where there is the means of reversing the driving power; or the cleaning is effected without reversing, by throwing in while at full speed one or two cwts. of some brittle and dry material.

The $4 \frac{1}{2} \mathrm{ft}$. machine is capable of pulverising 5 to 15 tons of material per hour, according to the nature of the materials and the degree of fineness to which they are reduced; the amount of power required to drive the machine, which varies with different materials, is from 10 to 25 horse power. 
The mineral disintegrator is in successful use for pulverising a variety of different materials. In a less perfected form it has been several years in use for breaking up the cakes or conglomerated lumps of artificial manures after manufacture, and the bone asb and mineral phosphate from which they are made, reducing the product to a fine granular powder for sowing with the grain. It is also used for pulverising the coal and pitch from which artificial fuel is manufactured, and the hard rock asphalte employed for making roadways; and in these applications the peculiar action of the disintegrator is found particularly advantageous in bleaking up and pulverising the material by free blows, avoiding all the compressing action that occurs in the processes of grinding or crushing, which canses a tendency to clog the machines on account of the tough nature of some of the material operated upon. Raw clay for the manufacture of fire-bricks and sanitary tubes is prepared by the disintegrator, both dry as dug from the pit, and in the wet and weathered state; and burnt brick and earthenware are pulverisel by it for mixing with the clay. The ores of zinc, namely blende and calamine, are broken up for smelting, and other hard materials, such as gold quartz; also cattle food, such as locust beans, oil cake, \&c.

The disintegrator is also very efficient for mixing as well as for pulverising; the action of the successive cages of beaters revolving in opposite directions and expelling the mixed materials all round the circumference is a remarkably perfect means of effecting a thorough mixture. It is in extensive use in sugar factories for mixing the various shades of moist sugars into the required uniform hue; and it is also used for mixing the lime, sand, and hair, for mortar making, in which application it has the advantage when driven at a moderate speed of being free from the objection of reducing the sand to fine powder, as is done by edge-runner mills. A further extension of its application may perhaps be effected for finely granulating metals in a melted state, by employing a machine revolving horizontally, without an external casing, so as to project the drops of melted metal from the 
circumference and allow them to fall into water, for the production of lead shot or other purposes.

Mr. CARR showed models of the disintegrating flour mill and the mineral disintegrator, with a variety of specimens of different materials pulverised by the latter, including blende and calamine, fireclays, potsherds from glassworks, \&c.; also samples of the wheat meal as produced by the disintegrating flour mill, and of the fine flour, semolina, and bran, when separated from the meal by the ordinary bolting or dressing machines. In describing the models he drew attention to the circumstance that, as the two dises with their respective cages of beaters revolved quite clear and independent of each other, there was no friction between them, and the only friction to be overcome in the machine was simply that of the bearings of the two shafts; and that, in consequence of the dises revolving in opposite directions, each particle of the material passing through the machine enconntered the successive beaters at double the velocity at which they were actually running, being struck by them alternately in opposite directions. The disintegrating flour mill at present in use, as represented by the model exhibited, contained fourteen cages of beaters and as many as 1000 beaters in all, of 8 inches length; but that number of beaters and also their length were found to be more than was requisite, causing the loss of power in churning the air within the machine to be greatly increased beyond the amount that was unavoidable; this loss he believed would now be materially diminished by shortening the beaters and so bringing the two discs closer together, and he expected it would be found possible to bring them very much closer still, and thereby further to reduce this loss of power. With the beaters 8 inches long the strain produced npon them by the centrifugal force when the discs were revolving at a high 
velocity was so great that it was necessary to tie their outer ends together by rings connecting all the beaters in each circle; these rings were of crucible steel, and were made in the same way as weldless tyres, each being made originally broad enough on the circumference to allow of dividing it into three rings of the same dimensions. At the speed originally intended of 800 revolutions per minute, the tensile strain produced upon the outermost ring by the centrifugal force was calculated to amount to nearly 10 tons per square inch; but 400 revolutions per minute were now found by experience to be ample for the production of flour, with dises of 7 feet diameter; at that speed he believed any individnal grain of wheat was only a fraction of a second in passing entirely through the mill and issuing in the state of meal. Instead of the fourteen cages of beaters originally employed, eleven were now found to be quite sufficient, and the beaters were placed rather further apart, 2 inches clearance being allowed them in every direction, instead of the original $1 \frac{1}{2}$ inches; in the mineral disintegrator they were sometimes put as far as $4 \frac{1}{2}$ inches apart, and this increased distance was not found at all to diminish the degree of fineness to which the minerals were pulverised. In the disintegrating flour mill duplicate driving pulleys on the shaft of each disc were provided for the purpose of accommodating the straps to any situation in which the machine might have to work; when the countershaft driving the machine was situated at a lower level, it would generally be more convenient to have the straps upon the overhung pulleys at the ends of the disc-shafts; but when the driving countershaft was overhead, the straps could go round the inner pulleys, which was preferable; in either case one of the straps was of course required to be crossed and the other open, for driving the two discs in contrary directions.

The President enquired how large were the pieces of material that were supplied into the mineral disintegrator, and what was the power required to work the machine in pulverising any of the minerals of which specimens were exhibited.

Mr. Carr replied that the size of the pieces of material put into the machine depended upon their hardness and upon the strength of 
the machine employed for the work. The hardest material he had yet tried to pulverise was potsherds from the large pots employed in melting the materials for glass making; when these pots became cracked in working, they were rendered useless, and had to be broken up and pulverised for moulding with fresh raw fireclay into new pots. The potsherds were about as hard as granite, and very tough, and were therefore supplied into the machine in pieces not larger than the specimen exhibited, about 2 or 3 inches cube; and they were completely pulverised in once passing through the machine, as shown in the specimen exhibited of the powdered material produced. In pulverising zinc ore, a disintegrating machine of 4 feet 6 inches diameter driven by an engine of 15 horse power was found to pulverise 15 tons per hour to a granular powder of an exceedingly fine and uniform grain.

Mr. E. H. Carbutr mentioned that the Bradford Flour Mill Co. had one of the disintegrating flour mills, which had now been at work there about three months, and be believed they were very well satisfied with it. A certain proportion of the flour produced, after it had been screened, had to be passed through dressing stones for grinding it fine, as named in the paper; and he had also noticed that the bran had a good deal of flour adhering to it, and would therefore have to be put through the machine again in order to separate this. Some difficulty had been experienced in keeping the bearings of the machine cool, and it had been necessary to take off the top brasses and run water over the bearings while working, in order to cool them sufficiently.

Mr. CARR said it was only while the machines were new that the bearings were liable to heat, and they speedily improved in that respect. There was always flour adhering to the bran first delivered from the machine, as seen in the sample exhibited of the bran; this flour was separated from the bran by passing it a second time through the machine, by which the bran was thoroughly cleaned and the flour obtained separate from it by bolting, as shown in the second samples exhibited of the bran and of the flour. The semolina produced by the disintegrating flour mill employed at Messrs. Gibson and Walker's mill at Edinburgh, after separation from the fine flour 
and winnowing free from finely powdered bran, was also ground into fine by two or three pairs of millstones, and was mixed by bolting with the fine flour first produced.

Mr. J. H. Perks enquired what amonnt of applied power was utilised in working the disintegrating flour mill, and what was found to be the loss of power caused by the resistance of the air; he suggested that this loss might partly be obviated, as in the case of a centrifugal fan, by putting the external casing close enough to the circumference of the dises, so that the air should be carried round inside the casing by their revolution.

Mr. F. J. Bramweld said that on the occasion of the British Association Meeting in Edinburgh last August he had had an opportunity of seeing the disintegrator at work at Messrs. Gibson and Walker's Flour Mill, where the greatest facilities had been afforded by the proprietors for investigating its action, the whole of the other machines in one section of their large mill being stopped while indicator diagrams were taken by Mr. Edward Easton and himself. This section of the mill was driven by a compound-cylinder engine, which also drove a large number of other machines; all that it was possible to throw out of gear was disconnected, but nevertheless there was a great amount of shafting which could not be uncoupled. The disintegrator was therefore disconnected in the first instance, and the power required to drive the shafting alone was thus ascertained; and this power to overcome the shafting friction was deducted from the indications obtained in the subsequent experiments with the disintegrator. On considering the question of the power required to set the air in the disintegrator in motion, it had occurrred to him that the machine must act in a very different way from the action of a fan, because of the two sets of cages running in opposite directions, and thus producing conflicting motions in the air ; so that whether the casing of the machine were closed or not closed, much power must necessarily be consumed in this conflict. Although the whole quantity of air contained within the machine, which had beaters 8 inches long, weighed only about 2 lbs., yet the power expended in simply 
agitating that weight of air in the manner in which it was dealt with by the machine turned out to be very great. In order to ascertain correctly the power absorbed by this action upon the air, he had had the machine driven at the usual speed of about 400 revolutions per minute, pulverising 15 quarters of wheat per hour, and had found the power then consumed was 123 indicated horse. The machine was next made to run perfectly empty at the same speed, and the power consumed was found to be 63 horse. The driving strap of one of the dises was then thrown off, and the two dises, having been securely lashed together with cords round the beaters, were driven at the same speed as before but both in the same direction, so that the whole machine revolved like an ordinary centrifugal fan, without the reversed action of the second set of beaters; the power then consumed in driving the machine was only 19 horse. It therefore appeared that the difference between 63 and 19 horse power, namely 44 horse power, was expended in simply churning the air that was in the machine; this was certainly a very interesting and remarkable result.

An experiment was then made to ascertain the power required to pulverise 20 quarters of wheat per hour, which was found to be 145 horse; and as in the previous experiment 15 quarters per hour had taken 123 horse, the difference between these two results gave 22 horse power for pulverising the additional 5 quarters of wheat per hour, or $4 \frac{1}{2}$ horse power per quarter of wheat ground per hour; the pulverising of the 15 quarters per hour had consumed 123 less 63 , or 60 horse power, being only 4 horse power per quarter. These amounts were so very small, compared with the power required in grinding by ordinary millstones, that there could be no doubt about the largo saving attendant on the use of the disintegrator, even after deducting the power shown to be lost by agitating the air in the machine, and after also allowing for the fact that the machine did not complete the grinding of the whole of the flour at one operation, but produced a large proportion of semolina which required to be ground afterwards between stones. There was also a very important saring in the first cost of the machine, in the space occupied by it, and in the labour and 
maintenance attendant upon its working. In all the experiments he had made with the machine, the bearings worked perfectly well and continued quite cool.

In corroboration of the statement respecting the large amount of power required to churn the air, he might mention he had been informed that when the machine was first put to work it had been driven at 700 revolutions per minute, and in running empty at that speed without any grain it had absorbed the whole power of the engine, and the temperature of the external casing had risen to about $110^{\circ}$ Fahr. in two or three minutes, notwithstanding that there was a free passage for the air through the machine. He was glad to see an endeavour was now being made to remove the difficulty arising from the resistance of the air by the very simple expedient of diminishing the width of the machine. An idea that had occurred to him for obviating that difficulty had been to work the machine in a partial vacuum, or even in an atmosphere of hydrogen which would have only one fourteenth of the weight of the air; but bringing the discs closer together would clearly have the same effect of diminishing the total weight of air contained within the machine. No doubt this was the simplest way of removing the difficulty, and it was attended with the advantage that, in consequence of the beaters being made so much shorter, the strengthening rings connecting their outer extremities could then be got rid of, thereby simplifying the construction of the machine in the manner shown in Fig. 10. He enquired whether any of these narrower machines had yet been got to work; and he suggested that it would be of much interest if the same experiments which he had tried on the present wide machine were repeated on the narrower one, so as to ascertain to what extent the loss of power due to the churning of the air was diminished by bringing the discs closer together.

He had first seen the disintegrating machine some years ago, used for pulverising potsherds, and had been very much struck by the principle of its action, which appeared to be perfectly new. He did not know of any other machine for pulverising materials where the only abutment against which the machine acted was that presented by the inertia of the particles of the material operated 
upon; in ordinary millstones, edge runners, rumblers, stampers, or crushers, and he believed in all other contrivances by which materials were reduced to powder, an abutment was provided by one part of the machine, against which the material was pressed by some other part of the machine, and the material was crushed or pulverised by coming between these two parts. In the disintegrator however the whole force of the machine was expended directly upon the material itself, which was knocked about right and left with a succession of rapid and violent blows until it was completely pulverised. This construction of machine appeared to him to be eminently satisfactory for general adoption on account of its compactness and durability; and an important practical advantage attending its use was that its efficiency in working was not impaired by wear, as was the case with most other machines; the beaters might decrease in size until they actually broke, and yet so long as they remained unbroken the extent of their wear did not signify, and they would still do their work as effectually when worn as when new, because they had only to strike blows upon the material, which they could do equally well, however much they might be reduced.

Mr. CARR said several of the narrower flour mills, intended to diminish the loss of power from the resistance of the air, were now in course of construction, but they were not yet in operation. Although they would be considerably reduced in width, he considered their capacity would still be beyond the requirements of their working, in consequence of the very great speed at which the grain passed through the machine and issued in the form of meal.

Mr. F. A. CowPen observed that he had had one of the $3 \mathrm{ft} .6$ ins. machines in use and also one of $6 \mathrm{ft}$. 3 ins. diameter for artificial manures, and they had worked very satisfactorily. In the larger machine the two driving shafts were arranged independently and opposite each other, the same as in the disintegrating flour mill, instead of making one of them hollow and carrying the other through it, as in the drawings shown of the mineral disintegrator; the bearings were found to work cool, and there had not been any 
occasion for running water over them as previously referred to. The quantity of material broken up by the machines was certainly very great. When working on artificial manures they became clogged with the phosphate of lime, and to clean them he had tried putting some hard dry material through the machine, but this did not answer perfectly; he thought the best plan was simply reversing the machine, when the backs of the beaters became the fronts, and they were thus cleaned by the further working of the machine. There might possibly be a little trouble in reversing the machine for cleaning, unless there were the means of readily reversing the engine for the purpose; but however frequently the cleaning had to be done, any inconvenience attending the operation was far outweighed by the very large amount of work performed by the machine. The samples of flour he remembered seeing at the Oxford Meeting of the Royal Agricultural Society in 1870 from the disintegrating flour mill were not in so perfect a condition as the samples of flour now produced, which were very good, and much superior to those obtained from the earlier machines; he considered the new mill was an important improvement in grinding. One of the mineral disintegrators he understood was employed at Boston in the United States for breaking up Missouri Mountain iron ore, which was a tolerably tough material, but was well broken up by the machine. A suggestion occurred to him with regard to the beaters of the disintegrator, whether they would not be better to be made square instead of round, and to be fixed with their flat sides radial. With the present round beaters, if a particle of material struck them on either side of the centre line, it would glance off without receiving the full force of a direct blow, and the efficiency of the beaters would thus be diminished. Square beaters on the other hand would give a full blow upon all particles striking anywhere upon their flat faces; and he thought the effect would be that three or four of the rings of beaters in the flour mill might be dispensed with by the use of square beaters.

Mr. CARR said he had not tried the use of square beaters in the disintegrator, but although they were perhaps more correct in principle than the round bars, he did not think much advantage 
would be gained by their adoption, because there would still be the liability of the particles of material striking the corners of the bars instead of their flat faces; it was therefore preferable he thought to make the bars round, as the simplest and safest plan. A large number of the mineral disintegrators were now in use in America, where they had been introduced about five years ago.

Mr. F. W. WEBB enquired whether the disintegrator had been tried for breaking up a hard friable metal such as spiegeleisen. He considered it would be a very useful machine if it could break up spiegeleisen so as to supply it to the steel converters in the Bessemer process in a pulverised state, instead of having to melt it beforehand as was at present the case.

Mr. CARR believed the disintegrator had not yet been tried upon spiegeleisen; but zinc ore and crucibles for glass melting were readily pulverised by it, as shown by the specimens exhibited, and harder materials than the latter especially could scarcely be found among those which were required to be pulverised.

Mr. T. Grennwood observed that he had seen some of these machines at work in France two or three years ago in the neighbourhood of Paris, where they were used for breaking up a mixture of lime and clay for making the best roman cement. The material was found at that place as a natural mixture, and had to be ground very fine, after which it was made into bricks, burned, and then broken up and ground fine again, ready to be used as cement. The difficulty of pulverising the raw material in the first instance had previously been very great, as it was a damp and tough substance, greatly resembling putty in appearance and stickiness; he had brought a quantity of it over to Leeds and had tried crushing it with edge-rollers, but it stuck to the rollers and was very difficult to work in that way, while any preliminary process of drying to prepare it for crushing more readily would have added too much to the expense of the manufacture. The disintegrator he had seen at work in France certainly struck him as a very remarkable machine, and was the only machine he knew of that would successfully pulverise such a material as that cement. The raw material dug from the rock was broken into large lumps and thrown direct into the 
machine, which was about 4 feet diameter with four rings of beaters; it was driven at a great speed, and pulverised the material very completely.

Mr. CARR said the disintegrator had been four years in use in France, and there were now nearly a hundred of them at work in different parts of the Continent for a great variety of purposes.

Mr. A. PAGET remarked that with the disintegrating flour mill 15 quarters of wheat per hour was the smallest quantity which had been mentioned as being pulverised by the machine; and although for a mill in a large town such a rate of production would no doubt be requisite, he thought it would be far beyond the wants of an ordinary country mill. He enquired therefore whether a machine had been constructed suitable for prodncing a smaller quantity, and what was the smallest size of the disintegrator that had yet been made as a flour mill ; if it were intended to come into general use in country districts, he considered it would be requisite that the capacity and consequent power required should be much smaller than that of the machine of which the particulars had been given in the paper. If the mill were employed in producing a smaller quantity of flour than its size was capable of yielding, the proportionate loss of power by churning the air would of course be increased; and a smaller mill taking less power wonld therefore be desirable where the required production was necessarily limited to a small quantity only.

Mr. Carr replied that he was now making two flour mills of 5 feet diameter and containing only nime cages, and one of 7 feet diameter containing eleven cages, all with the bars greatly shortened, for the express purpose of diminishing the action upon the air and producing a smaller quantity of flour. It would not be advisable he considered to go much below 5 feet diameter for the flour mill, because the lineal speed of the outermost beaters could not be reduced without interfering with the efficiency of the machine for the production of flour, and therefore as the diameter was diminished the number of revolutions per minute had necessarily to be increased proportionately, which increased the difficulty of keeping the bearings cool. The only further means of adapting the mill to a smaller production of 
flour would be by still further shortening the beaters and bringing the dises still closer together, so as to reduce the capacity of the machine; and the power consumed in pulverising the grain would of course be diminished in proportion to the reduced production of flour and the smaller quantity of air churned in a narrower mill.

Mr. F. J. Bramwell mentioned that in the experiments he had made with the disintegrating flour mill at Edinburgh one of the points he had endeavoured to ascertain was the difference in the loss of power when the machine was driven empty at varying velocities. The lowest speed at which it had been possible to drive the engine steadily enough for arriving at any definite results was 12 revolutions per minute; and from the indicator diagrams taken at this and several higher speeds, up to the ordinary speed of running, it was found that the power uselessly expended in churning the air increased abont as the cube of the velocity, in accordance with theory. The experiments were all made under the same conditions, as to access and discharge of air, that prevailed in ordinary working.

Mr. E. A. CowPER observed that, although the closing of the external casing would of course not prevent the loss of power in agitating the air, because of the contrary motion of the two sets of cages, jet the amount of the loss he considered would be somewhat less when the casing was closed than when the air was free to escape from the circumference of the mill, because in the latter case a much larger quantity of air would be passing through the machine from the centre to the circumference. and undergoing the action of the beaters. The main point of importance in the closing of the casing seemed to him to be that the quantity of air passing through the machine should be limited to the least that was possible, only enough being allowed to pass through for keeping the machine and flour cool and dry during the working.

Mr. F. J. BRAmwell remarked that by the charning of the air within the machine a large amount of power was being converted into heat, and if there were not air enough allowed to pass off continuously from the machine he believed the meal would get so hot as to be prejudicial to the flour. 
Mr. SAmpson Lloyd said he had seen the mineral disintegrators at work at Swansea, pulverising small coal for the manufacture of artificial fuel, and also calamine or zinc ore, which was a very hard mineral. The only disadvantage in breaking up such hard minerals in the machine was that the beaters wore out in the course of a few weeks, and when one bar broke it was apt to break others out also; and in some cases he understood these breakages had been found so serious as almost to cause the machine to be abandoned. For softer materials the disintegrator answered very well; and it seemed to him a very excellent machine for all purposes of pulverising.

The President enquired what was usually found to be the durability of the beaters in the mineral disintegrators when employed in breaking up bard material, and whether they were made of steel in all the machines.

Mr. CARR replied that.in machines employed regularly every day in breaking up a hard material like calamine at the rate of 15 tons an hour the bars were found to last he believed about six or eight months before any breaking occurred, being made of steel, $1 \frac{1}{2}$ to 2 inches diameter according to the hardness of the material to be pulverised. At Messrs. Dillwyn's smelting works at Swansea, where six of the mineral disintegrators were now in constant use for breaking up zinc ores, the bars of some had been replaced several times, but this had not been considered any objection to the machines when the quantity of work performed by them was taken into account. Even when several of the bars had been broken out, no difference was perceptible in the fineness of the material pulverised, and the machine only required to be driven a little faster to compensate for the smaller number of bars; the sooner however they were replaced the better, as the balance and consequently the smooth working of the machine would otherwise be interfered with. When a bar broke within the machine, it had of course to fight its way out through the outer rings of beaters, and might perhaps break a few other bars in doing so, but this was not always the case. There were only four rings of beaters in the mineral disintegrators, and when any of the bars broke in the outside ring they were thrown off without damaging any of the others; it was only when a bar broke in one 
of the three inner cages that there was a possibility of its doing any injury in making its way out. In the disintegrating flour mill the bars were also made of steel, for the purpose of getting them as small and light as possible, on account of the very high speed at which the mill was driven.

The President remarked that the working capacity of the disintegrator appeared to depend upon the length of the beaters, the diameter of the machine being determined by the nature of the material to be pulverised or by the speed requisite for the purpose of effecting its disintegration by reason of its inertia; and he enquired whether in the disintegrating flour mill at Edinburgh, with beaters 8 inches long, a greater quantity than 20 quarters of wheat per hour had been pulverised, and what was considered the maximum result which a mill of that size would be able to produce.

Mr. CARR replied that 20 quarters of wheat per hour was the greatest quantity that had been pulverised by the mill at Edinburgh, but only because this produced as much flour as there was the means of dressing in the same time; the mill itself would no doubt be able to produce a much greater quantity, but the limit of its production had not been ascertained. So far as weight was concerned, the 20 quarters of wheat per hour amounted to less than $4 \frac{1}{2}$ tons, whilst the mineral disintegrator pulverised 15 tons of calamine per hour; but on account of the lightness of the flour it occupied a much greater bulk, and the flour mill at Edinburgh had therefore been made of the width shown, in order to ensure ample capacity for the required production, the circumstance not then being known that if the capacity were too great for the requirements so much power would be needlessly wasted in churning the air.

The President enquired whether supplying the grain more rapidly to the mill was found to cause a larger proportion of semolina to be produced, on account of the blows being softened by the striking surfaces beconing encumbered with the meal.

Mr. CARR replied that the proportion of semolina produced was not found to be affected by the quantity of grain passing through the machine; but it did depend on the speed at which the machine itself was driven, for the higher the speed the greater was the 
percentage of fine flour and the smaller the proportion of semolina; and this gave the means of altering the proportion by adopting a different speed for the machine.

Mr. T. GREenwood enquired whether in any of the disintegrating flour mills the speed had been ascertained at which the flour would become heated to an objectionable temperature.

Mr. CARR replied that a speed sufficient. to heat the flour injuriously had not been reached in any of the disintegrating flour mills, and the highest temperature to which the air had been heated in the mill, when running empty and at nearly double the speed now used, was only about $110^{\circ}$ Fahr., or little more than the temperature of a summer day in the sun; if the flour was at all damp, even that extent of heating would be beneficial for drying it and thereby rendering it less likely to go sour.

Mr. T. Grennwoon said that in grinding flour by means of flated steel rollers he understood the flour was not heated at all, and that consequently no fermentation took place and the flour would keep for a great length of time. At a large mill at Pesth in Austria, where 50 tons per week were being ground in that way, he had been shown flour which had been kept for ten years in a dry storehouse, and continued perfectly good on account of not having been heated; but he doubted whether any flour ground by stones would keep good so long. In that mill there was a series of about sixteen of the steel rollers placed one above another, and the whole of the grain passed alternately backwards and forwards between all of them in succession, making fifteen times of passing through; the effect was that the flour was not heated at all, as it was so short a time between the rollers, and was not subjected to the continued rubbing that it underwent between millstones.

Mr. CARR said the flour would not become discoloured by scorching at any temperature below $212^{\circ}$ Fahr., and there was therefore no risk of its being so injured if heated to $110^{\circ}$. With regard to fermentation, this arose not from the brief application of artificial warmth, but from the "sweating" resulting from the presence of too much moisture; and diminution of the moisture would therefore diminish the tendency to ferment. 
Mr. E. H. CARbutT observed that $110^{\circ}$ had been mentioned as the temperature to which the mill and casing were heated when the mill was running empty and was only churning the air; and the temperature would therefore evidently be considerably lower when the flour was passing through, because a portion of the heat would be carried off continuonsly by the flour.

Mr. CARR remarked that the small amount of moisture contained in the flour also rendered much of the heat latent by evaporation; and moreover, as the sheet-iron casing had time to accumulate the heat from the air, it was reasonable to suppose it would always be warmer than the flour, which was only so briefly exposed to the heating.

Mr. F. J. BrA MweLl observed that the introduction of Mr. Bovill's plan of aerating the flour by means of a fan blast, in grinding with millstones, had had for its object both to dry the flour by the current of air taking up the moisture, and also to keep the flour cool throughont its passage between the stones, and thereby prevent the liability of its becoming sour; and in the disintegrating flour mill the material was so thoroughly and freely knocked about, and was exposed all the time to such a strong current of air, that he thought a still better aeration and drying were obtained than were possible when the flour was confined between millstones. The temperature of the flour he believed was sensibly lower in the disintegrating flour mill, for on feeling it by hand as it came from that mill he had found it decidedly cool as compared with that nsually delivered from ordinary millstones.

The plan which had been referred to as in use at Pesth of grinding corn by means of steel rollers was known in England as Buchholz's plan, and was in use he believed in Liverpool. Another Pesth system of grinding was also in use at the North Shore Flour Mills, Liverpool, consisting of six pairs of stones with as many sets of intermediate dressing apparatus: the whole of the grain was passed through the first pair of stones, but was only very slightly ground by them, and then underwent a complete dressing, after which the residue was passed through the second pair, and so on throngh all the six pairs of stones, the dressing being 
repeated between each grinding; the semolina was got out in large quantities at about the third or fourth pair of stones. The results appeared to be highly satisfactory, notwithstanding that in this instance, owing to want of space, there were only six pairs of stones, instead of the nine pairs requisite for fully carrying out the system; and he had no doubt that when completely carried out on a large scale it would be still more successful. In the disintegrating flour mill however, equally advantageous results appeared to be obtained with simpler and less expensive machinery.

Mr. E. A. Cowper enquired whether the Hungarian plan of steaming the grain beforehand was used in the disintegrating mill at Edinburgh, and also whether it was crushed by rollers before entering the disintegrator.

Mr. F. J. Bramwel said that at the Edinburgh mill the grain was steamed and was also slightly crushed by running between rollers before passing through the mill.

The President considered there were many points of great interest connected with the disintegrator, both as to its theoretical action, and as to its practical applications, which were very numerous and would no doubt become still more extensive. One remarkable feature of the machine was that while it completely pulverised the material it also mixed the particles in a most thorough manner; and this mixing action would be very valuable in many metallurgical processes. He hoped shortly to make an application of it in that way for mixing hard anthracite with bituminous small coal, for the purpose of making coke in South Wales. He had himself seen some of the disintegrators at work, and had been much struck by the efficiency of their action.

He proposed a vote of thanks to Mr. Carr for his paper, which was passed.

The following paper was then read:- 


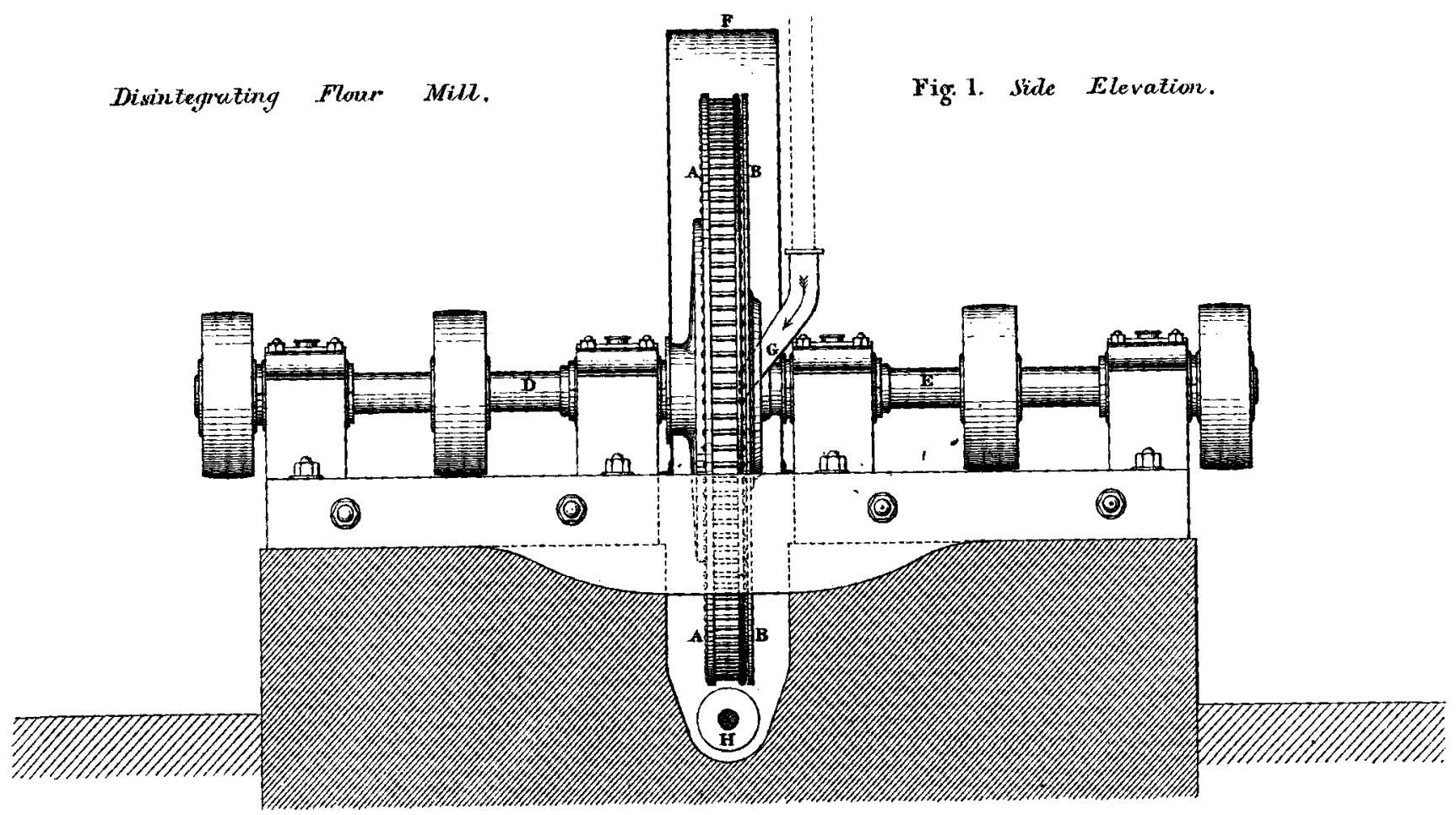

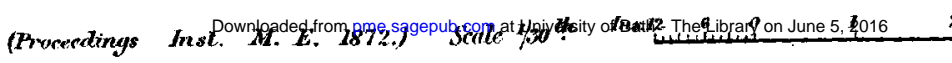
goroxt. 


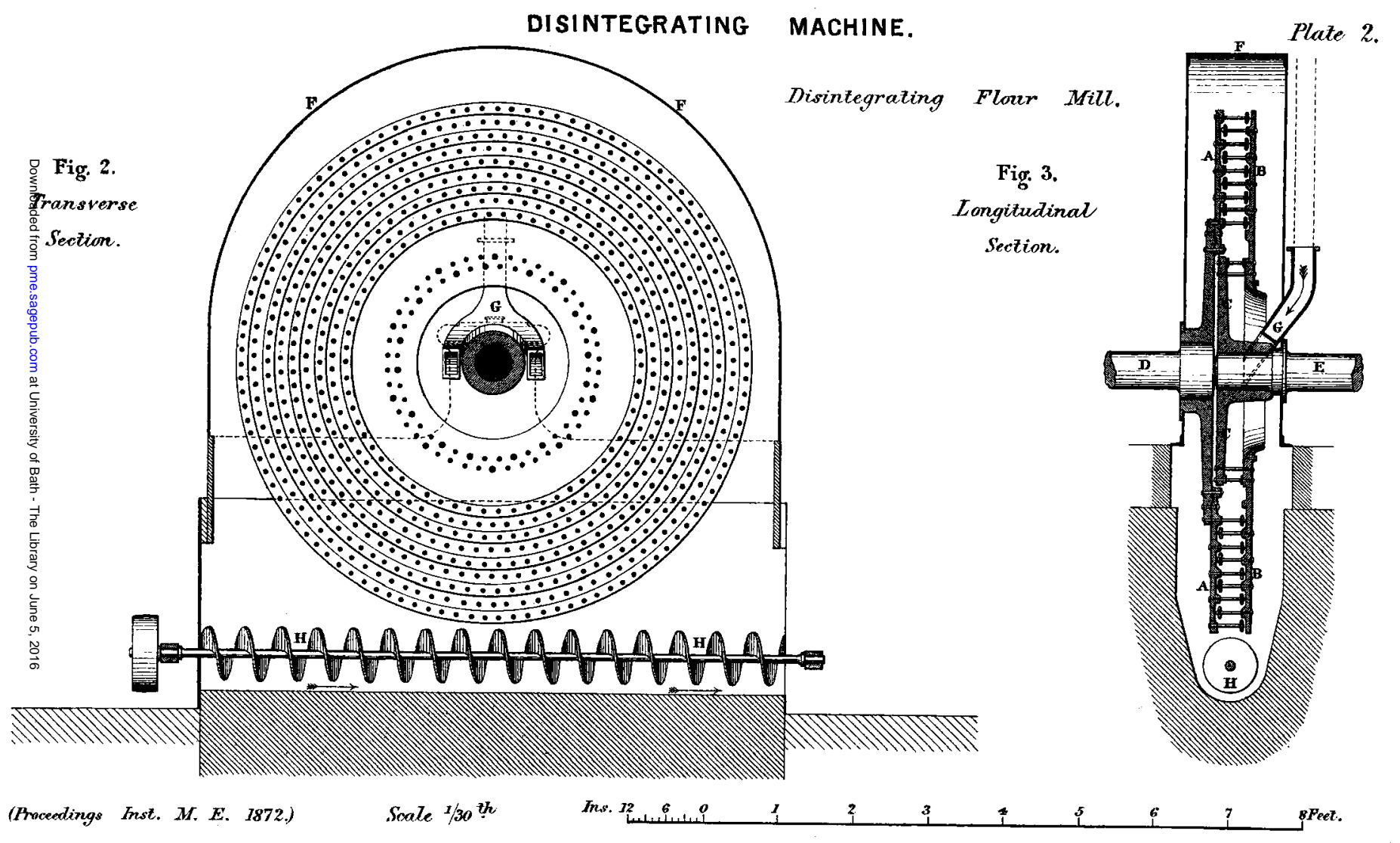




\section{DISINTEGRATING MACHINE.}

Plate 3.

Fig. 4. Transverse Section of Disintegrating Flour Mill,

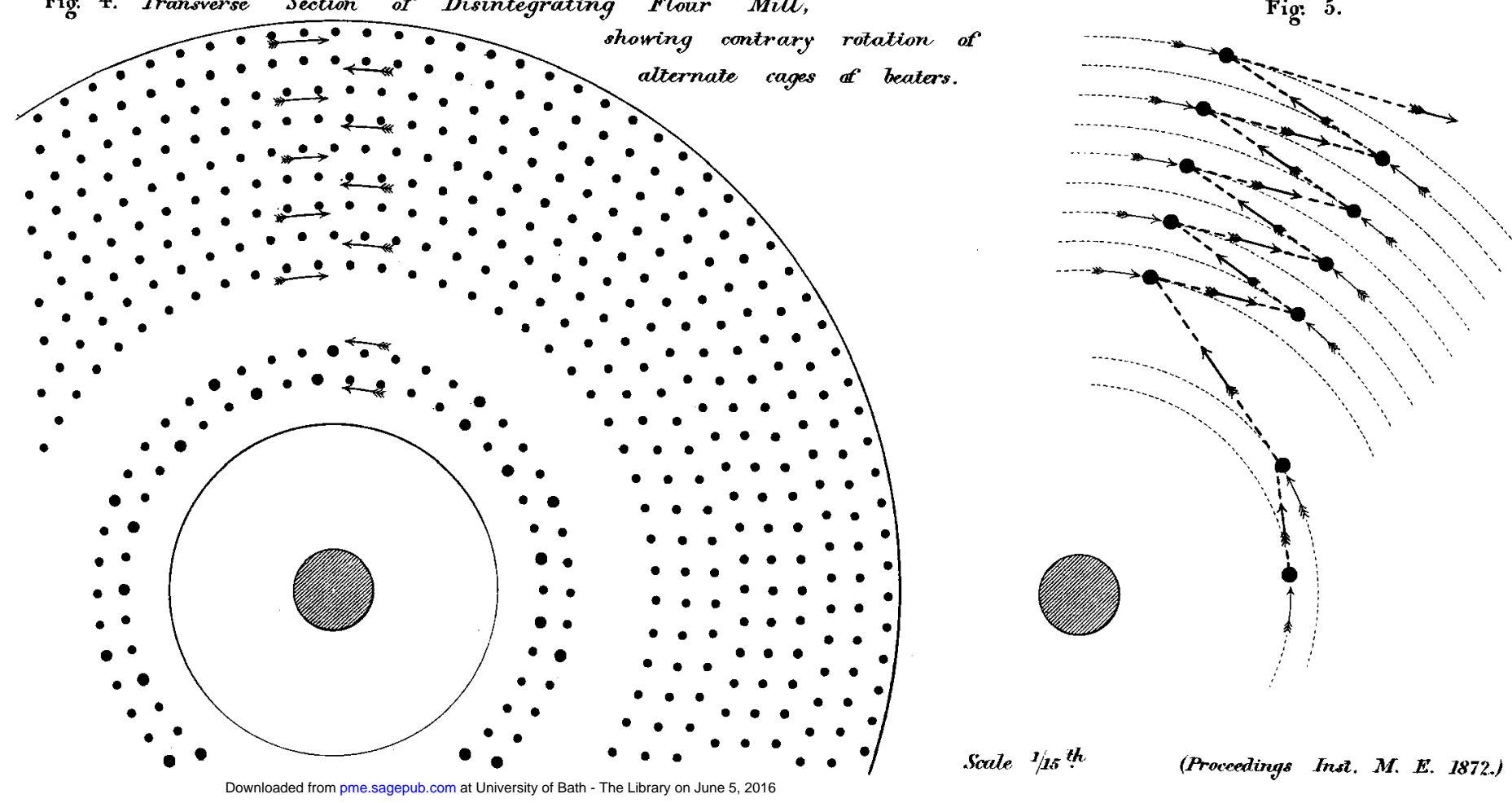


DSINTEGRATING MACHINE.

Antineral Disintegrator.

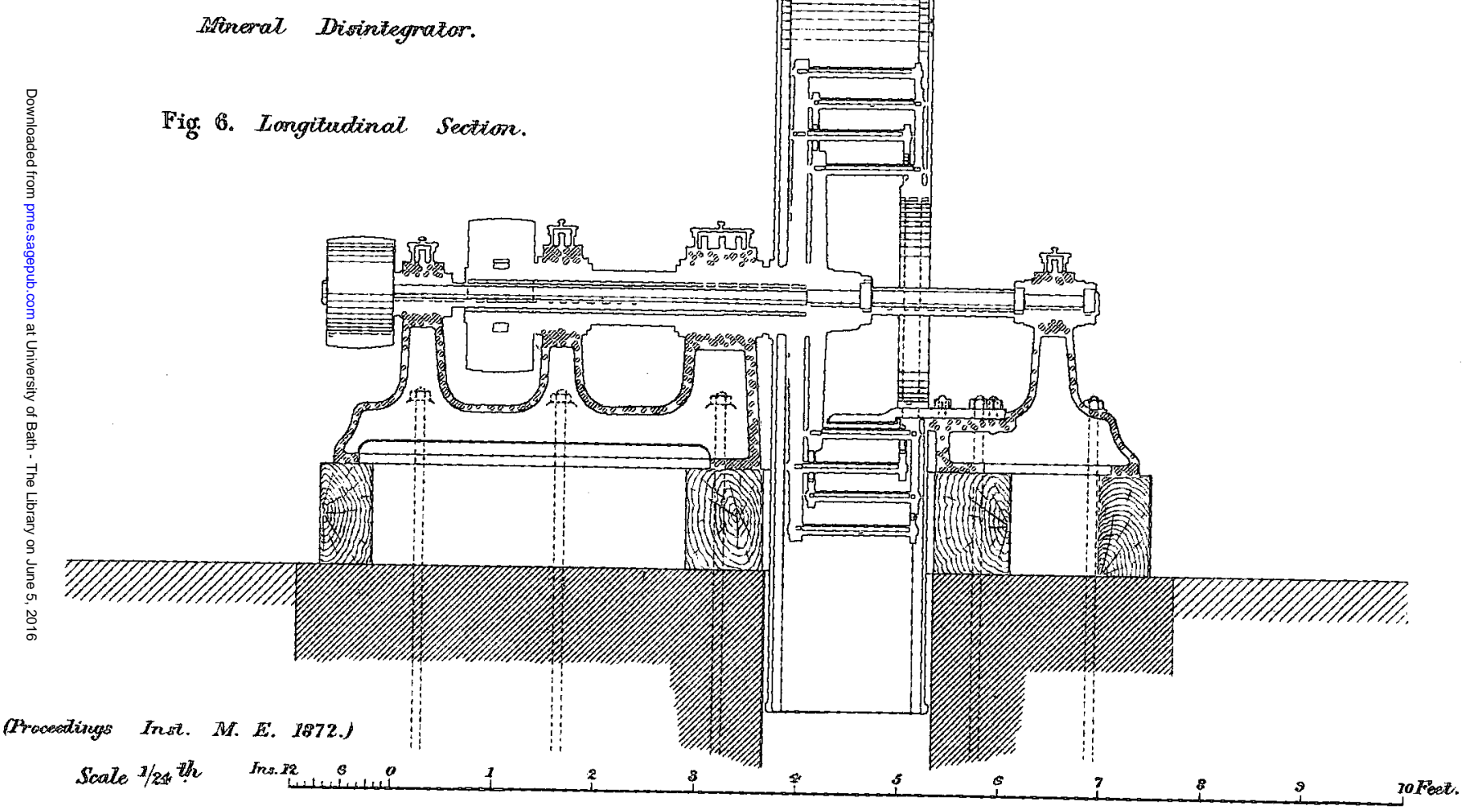




\section{DISINTEGRATING OAACHINE.}

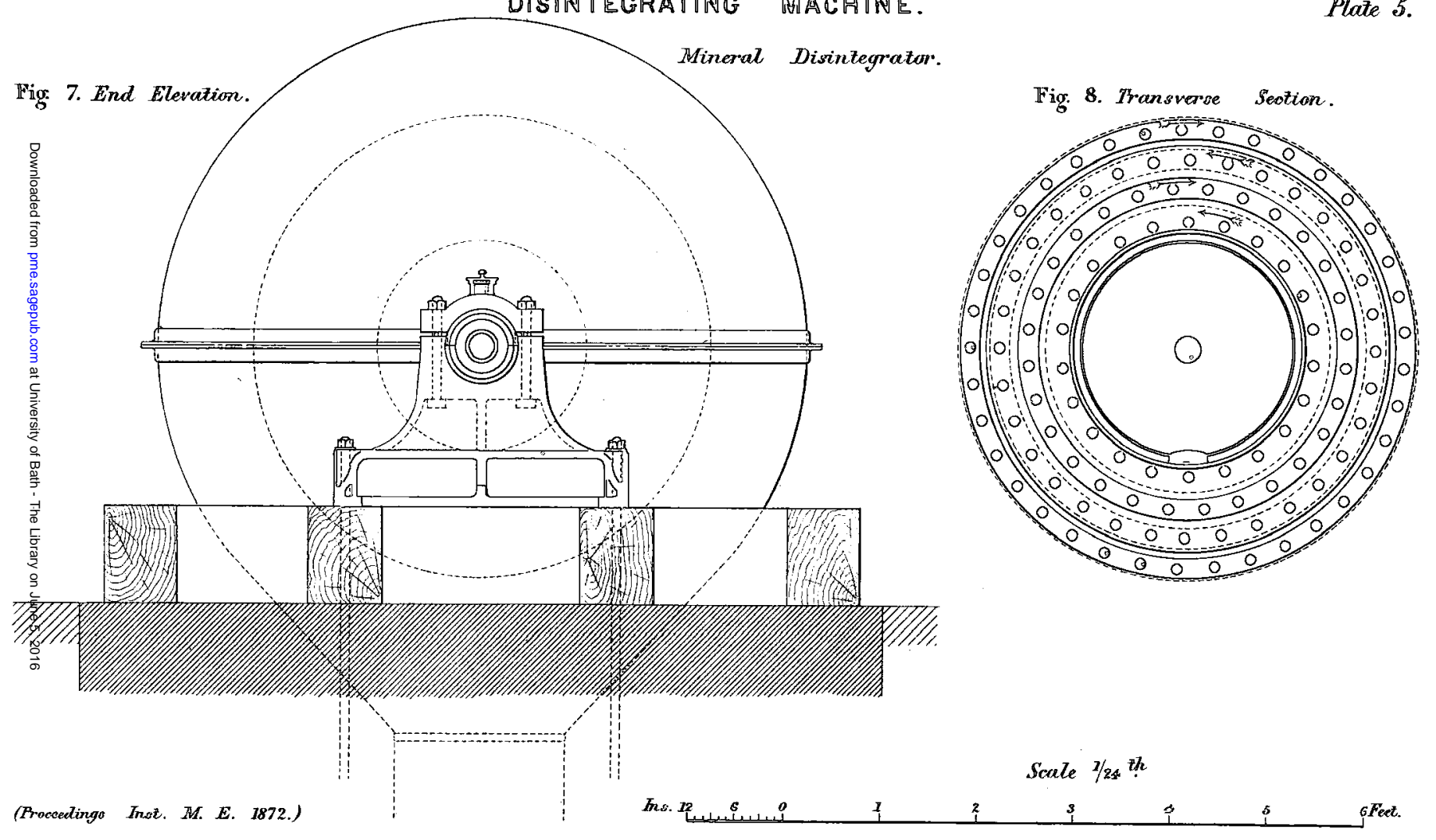




\section{DISINTEGRATING MACHIANE. IMIT G}

Fig. 9. Longitadinal Section of Disintegrating Flom, $1 / 1 /$.

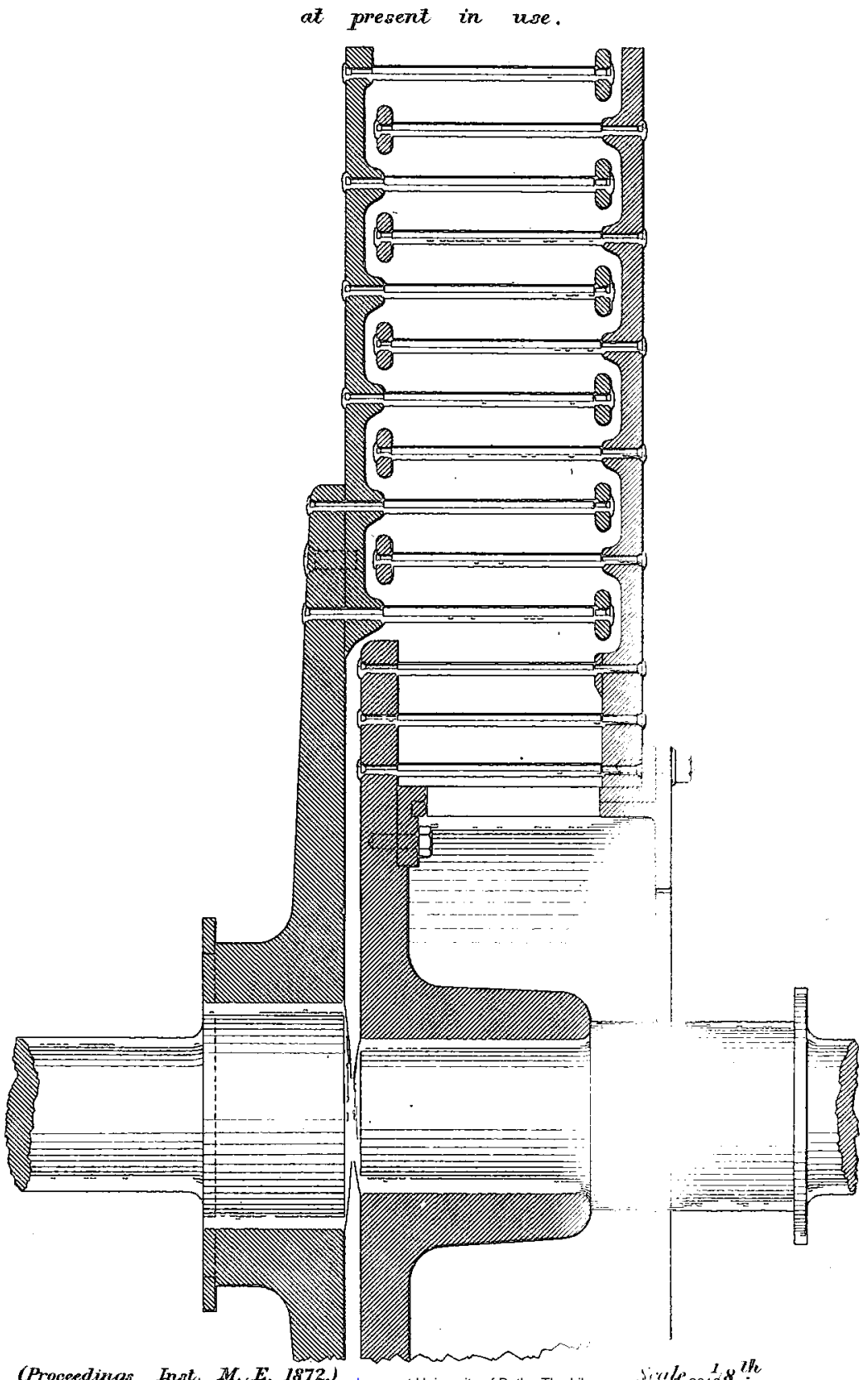




\section{DISINTEGRATING MACHINE. Plate 7.}

Fig: 10. Longltudinal Section of improved Disintegrating Flour Mill.

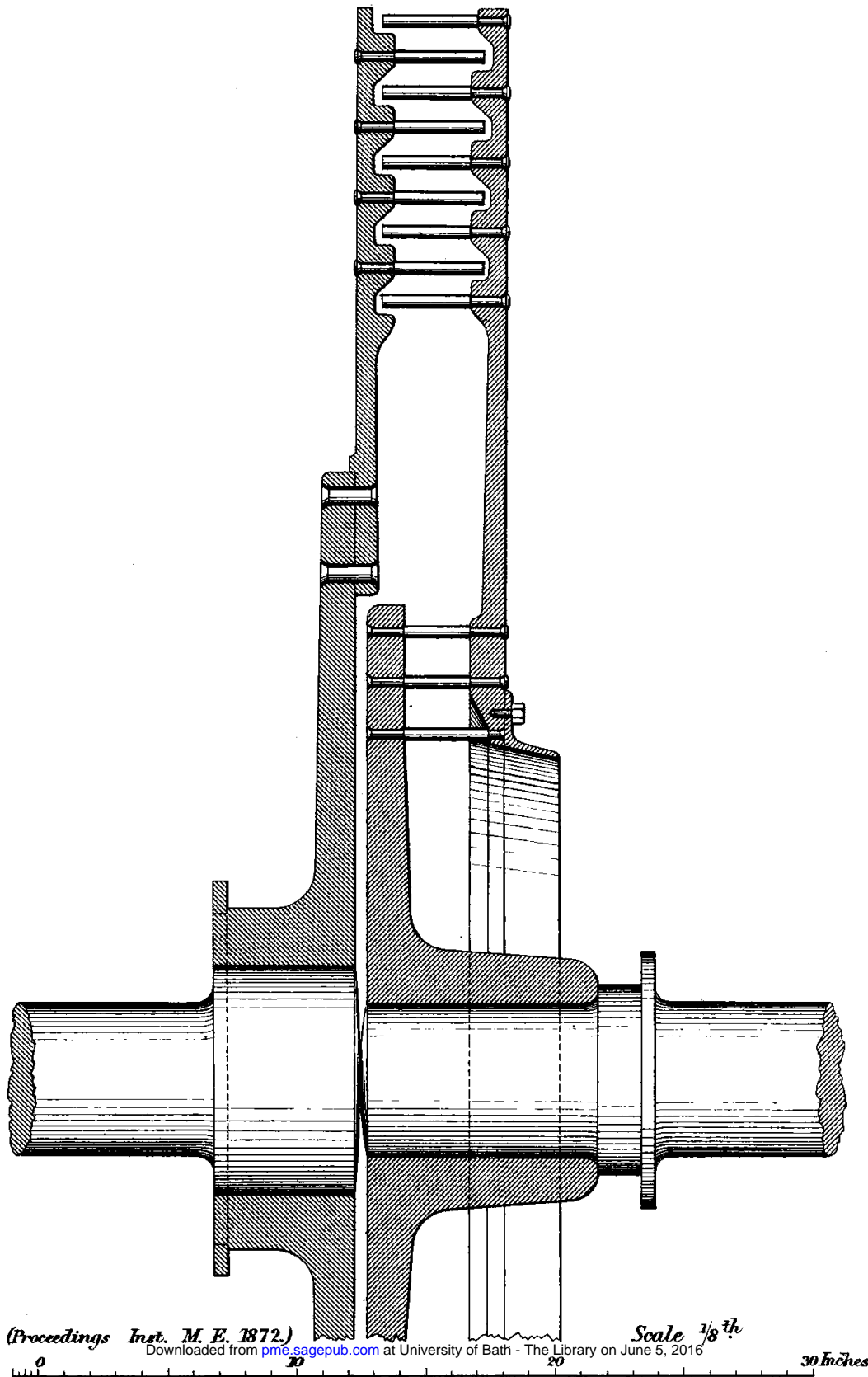

